\title{
Caracteristicas de los sangrados en niños con hemofilia en un centro de referencia en Colombia
}

\author{
Characteristics of bleeding in children with \\ hemophilia in a reference center in Colombia
}

Yadira Valderrama-Vargas ${ }^{1}$; Adriana Linares-Ballesteros ${ }^{1}$

\begin{abstract}
Forma de citar: Valderrama Vargas Y, Linares Ballesteros A. Características de los sangrados en niños con hemofilia en un centro de referencia en Colombia. Rev Univ Ind Santander Salud. 2018; 50(1): 19-26. doi: http://dx.doi.org/10.18273/revsal.v50n1-2018002 (c) (i)
\end{abstract}

\section{Resumen}

Introducción: La hemofilia es el trastorno hemorrágico congénito más frecuente en los varones, los pacientes con enfermedad severa pueden tener sangrados espontáneos que llevan a discapacidad. Objetivo: Describir las características de los sangrados y los aspectos clínicos y demográficos de un grupo de pacientes con diagnóstico de hemofilia en un centro de referencia de la ciudad de Bogotá. Materiales y métodos: Estudio descriptivo de serie de casos, en menores de 18 años con hemofilia, entre mayo 1 a diciembre 31 de 2014. Resultados: Se incluyeron 51 pacientes, 33/51 tuvieron sangrado, 109 sangrados, el sangrado más frecuente fue hemartrosis y en niños con anticuerpos inhibidores. 22 pacientes tenían hemofilia severa, 18 moderada y 11 leve. 18/22 pacientes severos sangraron y tuvieron 87 sangrados, 14/18 (77\%) pacientes con artropatía tuvieron sangrado, con 74 eventos en total, 19/33 pacientes sin artropatía tuvieron 35 eventos. El grupo con mayor frecuencia de sangrados tenía entre 5 y 9 años. De los 81 episodios traumáticos, la mayoría fueron jugando y haciendo deporte; Se presentaron 19 sangrados espontáneos y nueve relacionados con procedimientos. 84 sangrados fueron en temporada escolar y 25 en vacaciones. Al momento de los sangrados en 76 episodios estaban acompañados por padres o familiares y 69 episodios ocurrieron en el hogar (62,4\%). Conclusiones: Los sangrados fueron más frecuentes en niños con anticuerpos inhibidores, enfermedad severa, con artropatía, en temporada escolar y estando acompañados. No hubo mayor frecuencia de sangrados en aquellos con disfunción familiar.

Palabras clave: Hemofilia; Hemorragia; Niños; Cuidado del niño.

\begin{abstract}
Introduction: Hemophilia is the most common congenital hemorrhagic disorder in males. Patients with severe disease may have spontaneous bleeding leading to disability. Objective: To describe the bleedings and clinical features of children diagnosed with hemophilia and treated in a reference center in Bogotá. Methodology:Descriptive study of a case series of children (under 18 years) diagnosed with hemophilia, between May 1st and December

1. Clínica Infantil Colsubsidio. Bogotá D.C, Colombia.

Correspondencia: Yadira Valderrama Vargas. Dirección: Calle 67 No 10-67. Correo electrónico: yadira.valderrama@gmail.com.
\end{abstract} Teléfono: +573125858413 
31th 2014. Results: Fifty-one patients were included, 33/51 experienced 109 bleedings. Most common type were hemarthrosis and children with inhibitory antibodies had more bleeding episodes. Twenty-two patients had severe hemophilia, 18 moderate and 11 mild. In 18 of 22 severe patients, there were 87 bleeding episodes. 14/33 patients with arthropathy (77\%) experienced 74 bleeding events while the rest referred only 35. Patients between 5 to 9 years old had the highest frequency of bleeding. Among the 81 traumatic episodes, most frequent activities were playing and sport practicing; there were 19 spontaneous bleedings and nine were related to procedures. 89 bleeds occurred during school days and 25 on vacation. At the time of bleeding, in 76 episodes, patients were accompanied by family members. Conclusions: Bleeding was more frequent in children with severe disease, with arthropathy, during school time and while being accompanied. Bleedings did not occurred with a higher frequency in children with family dysfunction.

Keywords: Hemophilia; Bleeding; Child; Childcare.

\section{Introducción}

La hemofilia A (deficiencia del factor VIII de la coagulación) y la hemofilia B (deficiencia del factor IX de la coagulación) son el defecto de coagulación más frecuente de los varones. La hemofilia es una enfermedad huérfana, de muy baja prevalencia, de acuerdo al reporte de la Cuenta de Alto costo de 2016, en Colombia existen 2059 pacientes con hemofilia, 1705 con hemofilia A y 354 con hemofilia $\mathrm{B}^{1}$.

La principal manifestación clínica de la hemofilia es el sangrado, en los pacientes con enfermedad severa pueden ser espontáneos o traumáticos y en los pacientes con enfermedad leve y moderada es más frecuente que sean debido a traumas. Los episodios hemorrágicos pueden clasificarse de acuerdo a su localización en aquellos que ponen en riesgo la vida, como son los intracraneales, de cuello o gastrointestinales y graves cuando se presentan en articulaciones, músculos o mucosas de boca, nariz o sistema genitourinario ${ }^{2,3}$. Existen los sangrados menores como epistaxis, hematomas o equimosis de tejidos blandos, especialmente en extremidades, los cuales generalmente se controlan con medios físicos y no alteran el curso normal de la vida del paciente.

Se define evento hemorrágico como aquellos sangrados que requirieron tratamiento con sustitución de concentrado de factor VIII o IX ${ }^{4}$. Los pacientes con hemofilia severa pueden tener sangrados espontáneos, especialmente hemartrosis, que pueden llegar a ser tan frecuentes como 20 a 30 al año si no están en profilaxis con concentrado de factor VIII o IX ${ }^{5,6}$. La profilaxis es el tratamiento estándar de la hemofilia severa, la profilaxis protege de los sangrados espontáneos, y puede disminuir los traumáticos y las hemartrosis especialmente ${ }^{7-9}$. Los eventos de sangrado, modifican la normalidad en la vida del paciente, generan incapacidad para continuar con sus labores cotidianas, requieren de asistencia médica y es necesaria la administración de tratamiento específico para su adecuado control, la hemartrosis es el más frecuente y el que produce mayor disfunción en la calidad de vida del paciente ${ }^{10}$. El sangrado articular recurrente lleva a la artropatía hemofílica, es la principal complicación de los pacientes con enfermedad severa, se define como cambios degenerativos debido a episodios recurrentes hemorrágicos que pueden ser espontáneos o por traumatismos, con progresión hacía daño articular, que puede llevar a la limitación del movimiento y disminución de la independencia del individuo con discapacidad ${ }^{11}$.

La causa y frecuencia de los sangrados se conocen de los estudios clínicos para aprobación de los concentrados de coagulación, hay muy pocos estudios de descripción del tipo y circunstancias de los sangrados en pacientes con hemofilia. En Colombia no hay información sobre los sangrados y sus circunstancias en personas con hemofilia. El objetivo de este estudio es describir las características de los sangrados y los aspectos clínicos y demográficos de un grupo de pacientes con diagnóstico de hemofilia en un centro de referencia de la ciudad de Bogotá.

\section{Materiales y métodos}

Se realizó un estudio descriptivo de serie de casos. Se definió caso como el paciente menor de 18 años con diagnóstico de hemofilia A o B quien hacia parte del Programa de Atención Integral de Hemofilia de la Clínica Infantil Colsubsidio ubicada en Bogotá, Colombia. Se evaluaron variables demográficas y variables clínicas como el tipo de hemofilia, severidad, presencia de inhibidores, estado nutricional, presencia de artropatía hemofílica, características propias de los eventos hemorrágicos (tipo del evento, la localización del sangrado y los acompañantes durante el mismo), de la misma forma se midieron variables relacionadas con las actividades del paciente, entre las cuales se encontraban prácticas deportivas realizadas por el niño, tipo de 
colegio donde estudian, jornada escolar, y variables socioeconómicas y familiares que caracterizaban las condiciones de la vivienda, la funcionalidad familiar, usando la herramienta APGAR $^{12}$, los tipos de familia y las personas a cargo del menor.

Con el fin de determinar la presencia de evento hemorrágico como variable principal, éste se definió como aquel sangrado que requirió administración de concentrado de factor VIII o IX ${ }^{4}$, y artropatía como el compromiso articular definido por evaluación previa en la historia clínica, es definido por ortopedia o rehabilitación.

La recolección de la información se realizó mediante la aplicación de un instrumento estructurado por los autores, diligenciado durante las visitas de campo propias del programa en un periodo de ocho meses (entre mayo y diciembre de 2014). Este estudio tuvo en cuenta las recomendaciones de investigación en seres humanos referidas por la declaración de Helsinki y el informe Belmont. De la misma forma, se clasificó como una investigación sin riesgo de acuerdo a la resolución 8439 de 1993. En todos los casos se solicitó consentimiento informado firmado por los tutores de cada paciente y adicionalmente asentimiento para los mayores de ocho años.

Análisis estadístico: Se realizó un análisis descriptivo de la información. Para las variables cuantitativas se utilizaron medidas de tendencia central y de dispersión de acuerdo a la distribución de los datos. Las variables cualitativas fueron presentadas en frecuencias absolutas y relativas. La información fue procesada electrónicamente utilizando el paquete estadístico SPSS, la cual fue revisada seleccionando aleatoriamente el $10 \%$ de los instrumentos, los cuales fueron contrastados contra la base de datos, verificando la calidad de la información.

\section{Resultados}

Se analizaron 51 pacientes durante un periodo de ocho meses, $33(65 \%)$ tuvieron episodios de sangrado. La mediana de edad de todos los pacientes fue 11 años (RIQ 10), la mediana de edad de los pacientes que sangraron fue de nueve años (RIQ 9), la mayoría de los sangrados fue en los pacientes con enfermedad severa $(79,8 \%)$. En el grupo de edad entre cinco y nueve años, siete de nueve pacientes tuvieron sangrados $(77,8 \%)$ En la Tabla 1 se presentan las características clínicas de la población a estudio.
Tabla 1. Características clínicas de la población a estudio.

\begin{tabular}{|c|c|c|c|}
\hline \multirow[t]{2}{*}{ Características } & $\begin{array}{l}\text { Pacientes sin } \\
\text { sangrados }\end{array}$ & $\begin{array}{c}\text { Pacientes } \\
\text { con } \\
\text { sangrados }\end{array}$ & $\begin{array}{c}\text { Total de } \\
\text { pacientes }\end{array}$ \\
\hline & $\begin{array}{c}n=18 \\
(35,3 \%)\end{array}$ & $\begin{array}{c}n=33 \\
(64,7 \%)\end{array}$ & $\begin{array}{c}n=51 \\
(100 \%)\end{array}$ \\
\hline Hemofilia A/B & $15 / 3$ & $26 / 7$ & $41 / 10$ \\
\hline Hemofilia Leve & $6(54,5 \%)$ & $5(45,5 \%)$ & $11(21,6 \%)$ \\
\hline Hemofilia moderada & $8(44,4 \%)$ & $10(55,6 \%)$ & $18(35,3 \%)$ \\
\hline Hemofilia severa & $4(18,2 \%)$ & $18(81,2 \%)$ & $22(43,1 \%)$ \\
\hline $\begin{array}{l}\text { Pacientes con } \\
\text { inhibidores }\end{array}$ & 0 & $3(6 \%)$ & $3(6 \%)$ \\
\hline \multicolumn{4}{|l|}{ Grupos de edad } \\
\hline 1 a 4 años & $4(8 \%)$ & $10(19 \%)$ & $14(27 \%)$ \\
\hline 5-9 años & $2(4 \%)$ & $7(14 \%)$ & $9(18 \%)$ \\
\hline $10-14$ años & $7(14 \%)$ & $10(20 \%)$ & $17(34 \%)$ \\
\hline $\begin{array}{l}\text { Mayor o igual a } 15 \\
\text { años }\end{array}$ & $5(10 \%)$ & $6(12 \%)$ & $11(22 \%)$ \\
\hline \multicolumn{4}{|l|}{ Tipo de tratamiento } \\
\hline Pacientes en profilaxis & $5(20 \%)$ & $20(80 \%)$ & $25(49 \%)$ \\
\hline Pacientes a demanda & $13(50 \%)$ & $13(50 \%)$ & $26(51 \%)$ \\
\hline \multicolumn{4}{|l|}{ Estado nutricional } \\
\hline Eutrófico & $11(21 \%)$ & $17(33 \%)$ & $28(54 \%)$ \\
\hline Bajo peso & $5(10 \%)$ & $7(14 \%)$ & $12(24 \%)$ \\
\hline Sobrepeso & $2(4 \%)$ & $9(18 \%)$ & $11(22 \%)$ \\
\hline \multicolumn{4}{|l|}{$\begin{array}{l}\text { Compromiso } \\
\text { articular }\end{array}$} \\
\hline $\begin{array}{l}\text { Pacientes sin } \\
\text { artropatía }\end{array}$ & $14(27 \%)$ & $19(37 \%)$ & $33(64 \%)$ \\
\hline 1 articulación afectada & $1(2 \%)$ & $5(10 \%)$ & $6(12 \%)$ \\
\hline 2 a 4 articulaciones & $2(4 \%)$ & $8(16 \%)$ & $10(20 \%)$ \\
\hline $\begin{array}{l}5 \text { o más } \\
\text { articulaciones }\end{array}$ & $1(2 \%)$ & $1(2 \%)$ & $2(4 \%)$ \\
\hline $\begin{array}{l}\text { Número de } \\
\text { articulaciones } \\
\text { afectadas }\end{array}$ & 11 & 30 & 41 \\
\hline Codos & 3 & 10 & 13 \\
\hline Rodillas & 5 & 8 & 13 \\
\hline Tobillos & 3 & 12 & 15 \\
\hline \multicolumn{4}{|l|}{$\begin{array}{l}\text { Actividad deportiva } \\
\text { de los pacientes }\end{array}$} \\
\hline Práctica futbol & $8(16 \%)$ & $9(18 \%)$ & $17(33 \%)$ \\
\hline $\begin{array}{l}\text { Practica natación, } \\
\text { ciclismo, otros. }\end{array}$ & $8(16 \%)$ & $9(18 \%)$ & $17(33 \%)$ \\
\hline $\begin{array}{l}\text { No practica ningún } \\
\text { deporte }\end{array}$ & $2(4 \%)$ & $15(30 \%)$ & $17(33 \%)$ \\
\hline
\end{tabular}

Se presentaron en total 109 sangrados, con una mediana de sangrados de 2 (RIQ 2), el paciente con menos sangrados presentó 1 y el de mayor fue 16. Dos de los tres pacientes con anticuerpos inhibidores tuvieron 28 de los 109 sangrados $(25,7 \%)$.

En 81 episodios hemorrágicos la causa fue traumática $(74,3 \%)$, lo más frecuente en circunstancias relacionadas con deportes y juegos $27(25 \%)$. La 
mayoría de los episodios de sangrado se presentaron en época escolar, 81 (74\%). En 76 eventos de sangrado (70\%), los pacientes se encontraban acompañados de progenitores o familiares con quienes conviven y en 69 episodios $(62,4 \%)$ los sangrados fueron en el hogar. La gran mayoría de los sangrados se dieron en pacientes escolarizados $(n=27)$, tuvieron 91 sangrados $(83 \%)$. Se presentaron 74 episodios de sangrado en pacientes con artropatía $(68,9 \%)$. En la Tabla 2 se presenta la descripción de los eventos de sangrado.

Tabla 2. Descripción de los eventos hemorrágicos

\begin{tabular}{|c|c|}
\hline Características de los sangrados & $\begin{array}{c}\text { Total de } \\
\text { sangrados } \\
\mathbf{n}=109(\%)\end{array}$ \\
\hline Sangrados en pacientes severos con inhibidores & $30(27,5 \%)$ \\
\hline Sangrados en pacientes severos sin inhibidores & $57(52,3 \%)$ \\
\hline $\begin{array}{l}\text { Sangrados en pacientes leves y moderados sin } \\
\text { inhibidores }\end{array}$ & $22(20,2 \%)$ \\
\hline Sangrados en pacientes con artropatía & $74(68,9 \%)$ \\
\hline \multicolumn{2}{|l|}{ Localización del sangrado } \\
\hline Articular & $48(44 \%)$ \\
\hline Tejidos blandos & $31(28,4 \%)$ \\
\hline Mucosas & $15(13,7 \%)$ \\
\hline Musculares & $14(12,8 \%)$ \\
\hline SNC & $1(0,9 \%)$ \\
\hline \multicolumn{2}{|l|}{ Etiología del sangrado } \\
\hline Traumáticos & $81(74,3 \%)$ \\
\hline Espontáneos & $19(17,4 \%)$ \\
\hline Relacionados con procedimientos & $9(8,3 \%)$ \\
\hline \multicolumn{2}{|l|}{ Actividad realizada } \\
\hline Deportes y juegos & $27(24,8 \%)$ \\
\hline Actividades cotidianas & $24(22 \%)$ \\
\hline Espontáneo & $19(17,4 \%)$ \\
\hline Traumas contundentes & $15(13,8 \%)$ \\
\hline Conductas de riesgo & $13(12 \%)$ \\
\hline Procedimientos de cuidado de salud & $9(8,3 \%)$ \\
\hline Juegos de la infancia & $2(1,8 \%)$ \\
\hline
\end{tabular}

Acompañamiento en el momento del sangrado

Padres

$59(54 \%)$

Otros familiares

$17(16 \%)$

Amigos o compañeros

$25(23 \%)$

Sin acompañamiento

$8(7 \%)$

Lugar de presentación del sangrado

Domicilio $68(62 \%)$

Exteriores $25(23 \%)$

Plantel educativo $16(15 \%)$

Época del año de la presentación del sangrado

Temporada escolar $81(74 \%)$

Vacaciones $28(26 \%)$

22
Dentro de las variables demográficas se observó que 36 pacientes (76\%) procedían del área urbana y el 74\% vivían en estratos socioeconómicos 1 y 2 . El 51\% de los pacientes tiene vivienda propia y $36(76 \%)$ vivían en condiciones adecuadas que no sugerían hacinamiento. El $55 \%$ de la población está cursando grados de primaria y secundaria básica, 25 pacientes $(49 \%)$ estudia media jornada, el $60 \%$ se traslada al colegio caminando y 47 (93\%) gasta menos de 30 minutos para llegar. En el grupo observado, 19 de las madres (37\%) y 9 padres (17\%) tienen formación profesional, 16 madres (39\%) se dedican al cuidado del hogar.

Las variables familiares mostraron que 29 familias (56\%), corresponden al tipo nuclear completa. En 35 de las 51 familias analizadas $(68 \%)$ no hay disfunción familiar. En 31 pacientes $(69.8 \%)$, los padres consideran que los menores tienen actitudes de riesgo, en este grupo $22(70 \%)$ presentaron sangrados. En la Tabla 3 se presentan las características psico-sociales de los pacientes con y sin sangrados.

\section{Discusión}

La hemofilia es una enfermedad huérfana, de muy baja prevalencia $(4,5 \times 100.000$ habitantes $)$, de acuerdo al reporte de la Cuenta de Alto costo $2016^{1}$.

En el grupo de pacientes que se evaluó en este estudio se encuentra que casi dos terceras partes tuvieron sangrados. El grupo de pacientes con sangrados tenían una mediana de edad ligeramente menor que aquellos que no sangraron (9 vs 13 años); los pacientes entre 5 y 9 años fueron los que proporcionalmente tuvieron más sangrados (77,8\%), este grupo de edad es muy activo físicamente, a este grupo de edad pertenecen dos de los tres niños con inhibidores. Si se revisan los pacientes sin inhibidores se encuentra que el grupo de edad con mayor número de sangrados fue el de 10 a 14 años, muy similar a los reportado previamente en la literatura por Fisher, et al. que evaluaron los patrones de sangrado de pacientes con hemofilia sin inhibidores y en profilaxis y encontraron mayor número de sangrados en los pacientes entre 10 y 16 años $^{13}$. Los dos pacientes con mayor número de eventos de sangrado tenían anticuerpos inhibidores y uno de ellos artropatía, es sabido que los pacientes con inhibidores y artropatía tienen más hemartrosis, en un fenómeno de ciclo vicioso entre estas dos condiciones ${ }^{14-16}$.

De los pacientes que tuvieron sangrados, un poco más de la mayoría tenían hemofilia severa en contraste con aquellos que no sangraron $(81,2 \%$ vs $22 \%)$, todos aquellos con enfermedad severa estaban en profilaxis. En los pacientes de este estudio, se encontraron 18 
pacientes con artropatía (35,5\%) 12 de ellos tenían dos o más articulaciones comprometidas, nueve tuvieron sangrados y tres no sangraron, a pesar de tener este compromiso articular. El sitio de sangrado más frecuente fue articular, en $44 \%$ de los pacientes, seguido del tejido blando y muscular, esto es consistente con la literatura. En los pacientes severos en profilaxis, el sangrado más frecuente fue hemartrosis seguido por el de músculos y tejidos blandos, en la evaluación de sangrados de niños con hemofilia severa en profilaxis se encuentran las mismas causas por otros autores ${ }^{13,17}$. La artropatía puede contribuir a los sangrados especialmente en los pacientes con enfermedad severa, en este estudio se encontró artropatía en 15/22 pacientes $(68,2 \%)$ severos, se encontraron más eventos de sangrados en los pacientes con artropatía que en aquellos sin (74 vs 35), en estos pacientes cerca de la mitad de los sangrados fueron articulares $(48,6 \%)$. La explicación puede ser que la mayor proporción de pacientes iniciaron profilaxis por encima de los 2 años de edad; en Latinoamérica la frecuencia de artropatía se conoce poco, pero está entre $50 \%$ y $83 \%$ (18), otros países tiene una frecuencia similar a la hallada en este estudio (19). La gran mayoría de los sangrados se dieron en pacientes escolarizados, tuvieron 91 sangrados, 72 fueron traumáticos, 69 fueron en días escolares, esto difiere de los hallazgos de otros estudios, donde hubo mayor número de sangrados en la época de vacaciones, posiblemente porque son estudios en países con estaciones y es en verano cuando hay más eventos de sangrado, quizá esté en relación con las prácticas deportivas de verano de los niños que viven en países con estaciones, diferente al tipo de actividades de los pacientes en nuestro país durante las vacaciones (que puede equivaler al verano) $)^{13}$.

Respecto al estado nutricional, en nueve de los 11 pacientes $(81,8 \%)$ con sobrepeso se presentaron 26 sangrados, de los 28 pacientes eutróficos, 17 sangraron $(60 \%)$ con 51 eventos y de los 12 pacientes con bajo peso, siete sangraron $(58,3 \%)$ con 32 episodios, proporcionalmente hubo más sangrados en los niños con bajo peso, uno de esos pacientes tenía inhibidores y artropatía. Para pacientes que reciben profilaxis, Fischer, et al. encontraron $13 \%$ de sobrepeso, muy similar a lo hallado en este grupo $(12 \%)^{13}$. Se encontraron datos de estado nutricional en pacientes de Alemania en profilaxis y a demanda con $34 \%$ de sobrepeso/obesidad ${ }^{20}$ una evaluación de centro de Norte América mostró una prevalencia entre 20 a $22 \%$ de sobrepeso en niños y adolescentes respectivamente ${ }^{21}$. En nuestro estudio se encontró un $21,6 \%$, la menor proporción de sobrepeso de los pacientes en profilaxis quizá tenga que ver el seguimiento y acompañamiento más frecuente que tienen estos pacientes. El sobrepeso puede contribuir a agravar la artropatía ${ }^{20}$.

Tabla 3. Características psico-sociales de los pacientes sin y con sangrados.

\begin{tabular}{|c|c|c|}
\hline \multirow[t]{2}{*}{ Descripción } & $\begin{array}{c}\text { Pacientes sin } \\
\text { sangrados }\end{array}$ & $\begin{array}{c}\text { Pacientes con } \\
\text { sangrados }\end{array}$ \\
\hline & $n=18(35,3 \%)$ & $n=33(64,7 \%)$ \\
\hline \multicolumn{3}{|l|}{ Procedencia } \\
\hline Urbana & $14(27 \%)$ & $25(49 \%)$ \\
\hline Rural & $4(8 \%)$ & $8(16 \%)$ \\
\hline \multicolumn{3}{|l|}{ Características de los domicilios } \\
\hline Vivienda en Arriendo & $5(10 \%)$ & $18(36 \%)$ \\
\hline Vivienda Propia & $13(25 \%)$ & $15(29 \%)$ \\
\hline Sin Hacinamiento & $14(27 \%)$ & $25(49 \%)$ \\
\hline Hacinamiento & $4(8 \%)$ & $8(15 \%)$ \\
\hline $\begin{array}{l}\text { Distancia de la vivienda al centro } \\
\text { asistencial mayor de } 30 \mathrm{~min}\end{array}$ & $9(18 \%)$ & $22(43 \%)$ \\
\hline $\begin{array}{l}\text { Distancia de la vivienda al centro } \\
\text { asistencial menor a } 30 \mathrm{~min}\end{array}$ & $9(18 \%)$ & $11(22 \%)$ \\
\hline $\begin{array}{l}\text { Distancia del hogar al colegio } \\
\text { mayor de } 30 \text { minutos }\end{array}$ & $2(\%)$ & $1(\%)$ \\
\hline $\begin{array}{l}\text { Distancia del hogar al colegio } \\
\text { menor de } 30 \text { minutos }\end{array}$ & $13(\%)$ & $26(\%)$ \\
\hline \multicolumn{3}{|l|}{ Nivel académico pacientes } \\
\hline No escolarizado & $2(4 \%)$ & $6(12 \%)$ \\
\hline Pre-escolar y primaria & $8(16 \%)$ & $14(28 \%)$ \\
\hline Secundaria básica & $6(12 \%)$ & $11(22 \%)$ \\
\hline Educación media y técnica & $4(8 \%)$ & $2(4 \%)$ \\
\hline \multicolumn{3}{|l|}{ Forma de traslado al colegio } \\
\hline Transporte público & $1(2 \%)$ & $1(2 \%)$ \\
\hline Transporte privado & $3(6 \%)$ & $7(14 \%)$ \\
\hline Caminando & $11(\%)$ & $20(\%)$ \\
\hline No aplica & $3(6 \%)$ & $5(10 \%)$ \\
\hline \multicolumn{3}{|l|}{ Acompañamiento del menor } \\
\hline $\begin{array}{l}\text { Acompañamiento por cuidador } \\
\text { durante todo el día en hogar }\end{array}$ & $13(24 \%)$ & $30(60 \%)$ \\
\hline $\begin{array}{l}\text { No está acompañado gran parte } \\
\text { del día }\end{array}$ & $5(10 \%)$ & $3(6 \%)$ \\
\hline \multicolumn{3}{|l|}{ Persona a cargo del cuidado } \\
\hline $\begin{array}{l}\text { Principal cuidador del paciente } \\
\text { son los padres }\end{array}$ & $13(25 \%)$ & $23(45 \%)$ \\
\hline $\begin{array}{l}\text { Principal cuidador del paciente } \\
\text { son los abuelos }\end{array}$ & $2(4 \%)$ & $8(16 \%)$ \\
\hline Otros & $3(6 \%)$ & $2(4 \%)$ \\
\hline \multirow[t]{2}{*}{ Característica psicosocial } & $\begin{array}{c}\text { Pacientes sin } \\
\text { sangrados }\end{array}$ & $\begin{array}{c}\text { Pacientes con } \\
\text { sangrados }\end{array}$ \\
\hline & $n=18(35,3 \%)$ & $n=33(64,7 \%)$ \\
\hline Madre con educación superior & $10(20 \%)$ & $9(18 \%)$ \\
\hline Padre con educación superior & $6(12 \%)$ & $3(6 \%)$ \\
\hline Madre no trabaja, está en el hogar & $4(8 \%)$ & $12(24 \%)$ \\
\hline \multicolumn{3}{|l|}{ Diagnóstico por Psicología } \\
\hline Problemas paterno filiales & $12(24 \%)$ & $25(49 \%)$ \\
\hline Otras alteraciones & $1(2 \%)$ & $4(8 \%)$ \\
\hline No alteración & $5(10 \%)$ & $4(8 \%)$ \\
\hline
\end{tabular}


Los sangrados fueron más frecuente por mecanismos traumáticos, otros autores de evaluaciones de niños con hemofilia severa en profilaxis también encuentran que son más frecuentes los sangrados traumáticos ${ }^{13}$. Durante el seguimiento se tuvieron en cuenta situaciones como el día en que recibieron la profilaxis y el día del sangrado, se revisaron 51 sangrados, de los pacientes $\sin$ inhibidores y en profilaxis, en 16 eventos $(31,4 \%)$ el sangrado se presentó dentro de las 24 horas de administración del concentrado, en 15 eventos $(29,4 \%)$ el sangrado fue más de 24 horas después de la dosis de profilaxis, en 20 de ellos (39\%), después de 48 horas de la dosis de profilaxis. Se nota mayor relación entre el momento del sangrado y la última dosis de profilaxis más que la época del año, en una revisión de tendencias de sangrado para personas en profilaxis, se encontró que en menores de 18 años, hubo entre $22 \%$ y $38 \%$ de sangrados en personas que habían recibido profilaxis 48 horas y más antes ${ }^{13}$ : Kulkarni, et al. en la evaluación de efectividad de un nuevo concentrado de factor VIII, encuentra mayor número de sangrados en niños que habían recibido la dosis de profilaxis más de 48 horas antes $^{22}$. Es importante educar a la familia para hacer una revisión conjunta de los días de profilaxis y ajustarla a la actividad de los niños especialmente de aquellos con artropatía, tener en cuenta algunas circunstancias como la relación entre el día del sangrado y la administración de profilaxis.

Hay aspectos no evaluados en la literatura como el momento del sangrado y su relación con el acompañamiento por los cuidadores, ni donde se encontraban los niños en el momento de la hemorragia; en este estudio se presentaron más sangrados dentro del domicilio que en el colegio (68 vs 16), la gran mayoría traumáticos $(73,6 \%)$, de los 50 sangrados traumáticos en domicilio. En 33 pacientes (66\%), fueron por caídas o golpes con elementos contundentes del hogar, $6(8,8 \%)$ fueron relacionados con la canalización de venas para administración de profilaxis y los 11 restantes en otras circunstancias. La mayoría de los pacientes que tuvieron sangrados estaban acompañados por algún progenitor al momento del episodio $(n=59)$, comparado con aquellos que se encontraban solos $(\mathrm{n}=8)$.

En 17 pacientes se encuentra que tienen actividades de riesgo para presentar sangrados de acuerdo a la percepción de los cuidadores, en los pacientes sin inhibidores el grupo de edad con mayor número de sangrados fue el de 10 a 14 años, en algunos estudios los adolescentes tiene más sangrados posiblemente por los riesgos que asumen.
Dentro de los aspectos Psico-sociales, no hay en la literatura información sobre el tipo de vivienda y del colegio y la relación con sangrados, dos tercios de los pacientes vivían en situaciones de riesgo como tener vivienda en construcción, remodelación, ocho pacientes $(24,4 \%)$ vivían en condiciones de hacinamiento crítico.

Las variables familiares muestran que la gran mayoría tienen problemas paterno - filiales. Una evaluación de disfunción familiar en niños con hemofilia utilizando el Ápgar familiar, encontró que en niños con hemofilia la mitad tienen disfunción moderada, en nuestra evaluación solo una tercera parte tenían disfunción familiar utilizando este instrumento ${ }^{19}$. El $54,5 \%$ de familias son de tipo nuclear completa, los principales cuidadores son los progenitores, seis pacientes (18\%) no tienen presencia del padre en el hogar; $65 \%$ de las familias no tienen disfunción familiar.

\section{Conclusiones}

Los sangrados fueron más frecuentes en niños con anticuerpos inhibidores, enfermedad severa, con artropatía, en temporada escolar, estando en el hogar y acompañados por padres o familiares en primer grado. No hubo mayor frecuencia de sangrados en aquellos con disfunción familiar. Describir las situaciones y circunstancias de los niños con hemofilia que tienen sangrados, permitirá orientar las estrategias de seguimiento de los pacientes, especialmente de aquellos con enfermedad severa y con anticuerpos inhibidores. No se encontró mayor frecuencia de sangrados en niños con disfunción familiar. Una limitación de este estudio es el número de pacientes, sin embargo, es usual en enfermedad huérfana no tener grandes números de pacientes por la baja frecuencia de la condición.

\section{Agradecimientos}

A los pacientes y sus familias, que nos recibieron en sus hogares y participaron con la mejor disposición e interés posibles. A la Clínica Infantil Colsubsidio, al equipo del Programa de Atención Integral en Hemofilia, al Centro de Investigaciones de Colsubsidio, y al Dr. Carlos Castro, quienes nos apoyaron durante el desarrollo de esta investigación

\section{Consideraciones eticas}

De acuerdo con los principios establecidos en el reporte de Belmont y al artículo 10 de la Resolución 008430/93, este estudio se desarrolló conforme a los siguientes criterios: 
1- Se aplicaron los principios éticos básicos de respeto a las personas, beneficencia y justicia, analizando la información obtenida en las visitas de campo y entrevistas previo consentimiento informado verbal por parte de los pacientes y familias participantes.

2- Teniendo en cuenta que fue una investigación de tipo descriptivo, no se expuso a daño alguno a ninguno de los participantes, se protegió la confidencialidad y los resultados esperados de la investigación, están encaminados a generar bienestar a la población objetivo de este estudio.

3- La investigación fue desarrollada en una institución prestadora de salud de tercer nivel ampliamente reconocida por su compromiso social y altos estándares de calidad en la atención, previa autorización de su representante legal y la aprobación por el comité de ética en investigación institucional.

\section{Conflicto de interés}

No existe conflicto de interés.

\section{Referencias}

1. Cuenta de Alto Costo. Fondo Colombiano de Enfermedades de Alto Costo. Situación de la hemofilia en Colombia. Registro de información para el año 2016. Edición anual. Bogotá. Colombia. 2016.

2. Konkle BA, Huston H, Nakaya SF. Hemophilia A. En: Adam MP, Ardinger HH, Pagon RA, Wallace SE. GeneReviews. last revision: June 22, 2017. Seattle: University of Washington, Seattle; 1993-2017.

3. Srivastava A, Brewer AK, Mauser-Bunschoten EP, Key NS, Kitchen S, Llinas A, et al; Treatment guidelines working group on behalf of the world federation of hemophilia. Guidelines for the management of hemophilia. Haemophilia. 2013; p. 1-47.

4. Gringeri A, Lundin B, Von Mackensen S, Mantovani L, Mannucci pm, and The ESPRIT Study Group. A randomized clinical trial of prophylaxis in children with hemophilia A (the ESPRIT Study). J Thromb Haemost. 2011; 9(4): 700-710. DOI: 10.1111/j.15387836.2011.04214.x.

5. Acharya SS. Advances in hemophilia and the role of current and emerging prophylaxis. Am J Manag Care. 2016; 22(5 Suppl): 116-125.

6. Valentino LA, Mamonov V, Hellmann A, Quon DV, Chybicka A, Schroth P, et al. A randomized comparison of two prophylaxis regimens and a paired comparison of on-demand and prophylaxis treatments in hemophilia A management. J Thromb Haemost. 2012; 10(3): 359-367. DOI: 10.1111/j.1538-7836.2011.04611.x.

7. Manco-Johnson MJ, Abshire TC, Shapiro AD, Riske B, Hacker MR, Kilcoyne R, et al. Prophylaxis versus episodic treatment to prevent joint disease in boys with severe hemophilia. N Engl J Med. 2007; 357(6): 535-544.

8. Zhao Y, Xiao J, Yang R, Wu R, Hu Y, Beckmann $\mathrm{H}$, et al. Efficacy of standard prophylaxis versus on-demand treatment with bayer's sucroseformulated recombinant FVIII (rFVIII-FS) in chinese children with severe hemophilia A. Pediatr Hematol Oncol. 2017; 34(3): 138-148. DOI: 10.1080/08880018.2017.1313921.

9. Bertamino M, Riccardi M, Banov L, Svahn J, Molinari JC. Hemophilia care in the pediatric age. J. Clin. Med. 2017; 6(5): PiiE54. DOI:10.3390/ jcm6050054.

10. Rodríguez-Merchán EC. Articular bleeding in hemophilia. Cardiovasc Hematol Disord Drug Targets. 2016; 16(1): 21-24.

11. Melchiorre D, Manetti M, Matucci-Cerinic M. Pathophysiology of hemophilic arthropathy. J Clin Med. 2017; 25: 6(7). pii: E63. DOI: 10.3390/ jcm6070063

12. Bellón JA, Delgado A, Luna JD, Lardelli P. Validity and reliability of the family Apgar family function test. Aten Primaria. 1996; 18(6): 289-295.

13. Fischer K, Collins P, Bjorkman S, Blanchette V, Oh M, Fritsch S, et al. Trends in bleeding patterns during prophylaxis for severe haemophilia: observations from a series of prospective clinical trials. Haemophilia. 2011; 17(3): 433-438. DOI: 10.1111/j.1365-2516.2010.02450.x.

14. Morfini M, Haya S, Tagariello G, Pollman H, Quintana M, Siegmund B, et al. European Study on Orthopaedic Status of hemophilia patients with inhibitors. Haemophilia. 2007; 13: 606-612.

15. Dekoven M, Karkare S, Lee WC, Kelley LA, Cooper Dl, Pham H, et al. Impact of haemophilia with inhibitors on caregiver burden in the United States. Haemophilia. 2014; 20(6): 822-830. DOI: 10.1111/hae.12501.

16. Valentino LA. Assessing the benefits of FEIBA prophylaxis in haemophilia patients with inhibitors. Haemophilia. 2010; 16(2): 263-271. DOI: 10.1111/j.1365-2516.2009.02126.x.

17. Lentz SR, Cerqueira M, Janic D, Kempton C, Matytsina I, Misgav M, et al. Interim results from a large multinational extension trial (guardian $^{\mathrm{TM}} 2$ ) 
using turoctocog alfa for prophylaxis and treatment of bleeding in patients with severe haemophilia A. Haemophilia. 2016; 22(5): e445-449. DOI: 10.1111/ hae.12990.

18. Ozelo M, Villaca PR, Perez-Bianco R, Candela M, Garcia-chavez J, Moreno-Roodrigez B, Rodriguez MB, et al. Musculoskeletal evaluation in severe haemophilia A patients from Latinamerica. Haemophilia. 2014; 20(1): e63-e70. DOI: 10.1111/ hae.12316.

19. Fischer K, Astermark J, Van Der Bom G, Ljung R, Berntorp E, Grobbee DE, Van Den Berg HM. Prophylactic treatment for severe haemophilia: comparison of an intermediate-dose to a high-dose regimen. Haemophilia. 2002; 8(6): 753-760.

20. Hofstede Fg, Fijnvandraat K, Plug I, Kamphuisen P. W, Rosendaal Fr, Peters M. Obesity: a new disaster for haemophilic patients?A nationwide survey. Haemophilia. 2008;14:1035-1038.

21. Ullman M, Zhang QC, Brown D, Grant A, Soucie JM. Haemophilia treatment center network Investigators. Association of overweight and obesity with the use of self and home-based infusion therapy among haemophilic men. Hemophilia. 2014; 20(3): 340-348. DOI: 10.1111/hae.12303.

22. Kulkarni R, Karim FA, Glamocanin S, Janic D, Vdovin V, Ozelo $\mathrm{M}$, et al. Results from a large multinational cinical trial (guardian 3) using prophylactic treatment with turoctocog alfa in paediatric patients with severe haemophilia: safety, efficacy and pharmacokinetics. Haemophilia. 2013; 19(5): 698-705. DOI: 10.1111/hae.12165. 